mechanisms of auditory memory and other broad questions, but are lamentably vague about music itself. In two other chapters Clynes and his colleagues discuss the "essentic forms" of seven basic emotions, and the characteristic "pulse" by which a composer, they claim, hall-marks his compositions; but they fail to offer any empirical tests by which this claim could in principle be falsified.

Chapter 5, by Jackendoff and Lerdahl, is possibly the best in the book. These authors clearly recognize the central importance of structural concepts in the description of music, but even they omit to give any precise account of tonal relations, or to explain, for instance, the relation between the time signature of a classical composition and rhythmic structures of the individual bars. Of the remaining contributions, the most interesting are those of Diana Deutsch on the concept of Gestalt in music, of Sundberg on the expression of emotion in singing, and of Makeig on the theory of tonality. Makeig is familiar with the current theory of tonal relations but seems to be unaware of the insights expressed by the late Deryck Cooke in his classic book The Language of Music (Oxford University Press, 1959).

Elsewhere, Balzano offers a grouptheoretical account of "pitch sets", and rediscovers the three-dimensional tonal space first proposed by Longuet-Higgins in 1962 , but does not illustrate his proposals with any interesting musical examples. And Terhardt, an acknowledged authority on auditory pitch perception, proposes a musically outrageous "root sequence" for the first few bars of Tristan und Isolde, in which the augmented 4th ( $F$ to $B$ ) in the famous chord is implicitly misrepresented as a diminished 5th (E sharp to B). In all it seems that some neuropsychologists of music have much to learn before their ideas need to be taken very seriously by competent musicians.

Christopher Longuet-Higgins is Royal Society Research Professor in the Centre for Research on Perception and Cognition, University of Sussex.

\title{
Pascal's mind and Pascal's science
}

\section{A.W.F. Edwards}

Pascal: Adversary and Advocate. By Robert J. Nelson. Pp.286. ISBN 0-67465615-6. (Harvard University Press: 1982.) $\$ 22.50, £ 18$.

BLAISE Pascal was born in 1623 in Clermont, France. In 1631 his father Etienne Pascal (himself an able mathematician who gave his name to the "limacon of Pascal") moved to Paris in order to secure his son a better education. There, Etienne was one of the founders of Marin Mersenne's "Academy", the finest exchange of mathematical information in Europe at the time. To this informal academy he introduced his son at the age of 14 , and Blaise immediately put his new source of knowledge to good use, producing (at the age of 16) his Essay pour les Coniques based on the work of Desargues, another founder-member of the Academy.

In the succeeding years the young Pascal designed and had built the first mechanical adding machine and conducted experiments into the nature of a vacuum, but his chief scientific contribution was to lay the foundations of the theory of probability.

The fame of Pascal, however, rests only to a small degree on his scientific work. Both adversary and advocate, as the title of this book suggests, he employed his exceptional talents in the public pursuit of religious controversy (especially through the Lettres Provinciales) and the compilation of extensive notes (the Pensées) intended as the basis of $A n$

Apology for the Christian Religion which he did not live to complete. These works not only provide extraordinary insights into the human condition, but have had a great and lasting influence on French language and literature.

Yet his influence on probability was scarcely less; before his time, probability calculations amounted to no more than the enumeration of equally-probable outcomes in games of chance. Pascal introduced the important idea of manipulating expectations using recursively the fact that if expectations of gain $X$ and $Y$ are equally probable, the expectation is $1 / 2(X+Y)$. He also introduced the binomial distribution for equal chances and with its help, and that of mathematical induction applied to expectations, solved the Problem of Points for two players.

This problem was the topic of the correspondence between Pascal and Pierre de Fermat in the summer of 1654 which, together with Pascal's contemporary Traité du Triangle Arithmétique, includes not one but three solutions. Suppose two players stake equal money on being the first to win $n$ points in a game in which the winner of each point is determined by the toss of a coin, heads for one player and tails for the other. If such a game is interrupted when one player still lacks $a$ points and the other $b$, how should the stakes be divided between them?

On the evidence of the correspondence, Fermat and Pascal independently concluded that the problem could be solved by noting that at most $(a+b-1)$ more tosses will settle the game, and that if this number of tosses is imagined to have been made, the resulting $2^{a+b-1}$ possible games (each equally probable) may be classified according to the winner in each case, the stakes then being divided accordingly. Thus the real game, of indeterminate length, is embedded in an imaginary game of fixed length. Apart from this novel idea, however, such a solution by enumeration is straightforward, but Pascal offered both an independent method based on expectations which is valid for any number of players and, in the Traite $d u$ Triangle Arithmétique, the solution for two players in terms of the binomial distribution. This, now the standard solution, though derived from the solution by enumeration, was proved by Pascal by induction applied to expectations. The binomial distribution was not given algebraically but by reference to the "arithmetical triangle" of binomial coefficients, whose properties Pascal elaborated in his Traité (whence the name Pascal's Triangle).

An original result of Pascal's, given in the Traité, is the combinatorial proof of the fundamental relation

$$
{ }^{n+1} C_{r+1}={ }^{n} C_{r}+{ }^{n} C_{r+1},
$$

and this work also contains the first formal proof of the long-known binomial theorem for positive integral index.

Late in his annus mirabilis of 1654 Pascal suffered an intense religious episode which persuaded him to withdraw from society. Apart from some important work on the cycloid curve, he contributed nothing further of significance to mathematics. He died on 19th August 1662.

R.J. Nelson's book is an addition to the voluminous secondary literature on Pascal which will sit uneasily on a scientist's bookshelf. An examination of Pascal's psychology from the point of view of linguistic philosophy, it is itself written in a language that few will find easy. Thus:

To be sure, with its emphasis on the nonapodictic notion of figure and its composition in fragments, this most famous work of Pascal [the Pensées] does seem to lend itself to a theory of language skeptical of verbal mimesis, to a catachresis suggesting, at least to Marin, that Pascal views all language as aporetic, disjoined from the things, either objects or immutable ideas, that a mimological view of language posits as the referents or signifieds of words or signifiers.

It will be a while before the author's insights into the workings of Pascal's mind influence our understanding of Pascal's science, and that may be a pity, for Part I ("The Adversary") of his book is both interesting and accurate where it touches on scientific matters; but when the Two Cultures are divided by a common language it is inevitable.

A.W.F. Edwards is a Fellow of Gonville and Caius College and Reader in Mathematical Biology in the University of Cambridge. He has recently completed a book, Pascal's Arithmetical Triangle. 